\title{
Numerische Modellierung im Wasserbau
}

Im Zuge der Planung einer Anlage hat der Ingenieur Aufgaben zu lösen und verschiedene Entscheidungen zu treffen. Dafür stellen bereits ausgeführte Anlagen, die sich in ihrer Funktion bewährt haben, eine gute Basis dar. Speziell im Wasserbau sind wegen topographischer, geologischer und hydrologischer Randbedingungen Referenzbauwerke nur eingeschränkt zu finden, die dem Planer bei der Modellbildung helfen. Deshalb sind physikalische Modellversuche zur Abklärung von Detailfragen sehr vorteilhaft und helfen, negative Überraschungen beim Betrieb der Anlage zu vermeiden. Neben den Vorteilen der Versuche im Hinblick auf Anschaulichkeit, sind diese zeitaufwändig zu erstellen, damit kostenintensiv und beschränkt flexibel.

Eine exzellente zusätzliche Unterstützung bei der Modellbildung stellen numerische Verfahren dar. In kommerziell verfügbaren Programmen stehen mittlerweile robuste Algorithmen für die numerische Lösung der Differentialgleichungen und für die teilweise aufwändige räumliche Diskretisierung zur Verfügung. Die Anwendung der Programme erfordert neben dem Wissen über die numerischen Methoden, der Anwendungserfahrung, auch die Fähigkeit der geeigneten Interpretation der Ergebnisse. Eine große Rolle für die Qualität der Ergebnisse spielen dabei die Eingangskennwerte - und die Beurteilung dieser auf das Ergebnis und wie die Kennwerte ermittelt wurden. Eine Sensitivitätsuntersuchung mit der Variation der Eingangswerte ist dabei zu empfehlen, um auch eine Aussage über die Bandbreite der Ergebnisse zu erhalten. Ein so kalibriertes Modell ist dann für Variantenuntersuchungen ein sehr gut geeignetes Hilfsmittel.

Um für anstehende Berechnungen dem Ingenieur Hilfestellungen zu bieten, werden von Programmentwicklern Beispielrechnungen zur Verfügung gestellt. Diese dienen auch der Qualitätssicherung für Programmerweiterungen. Neben der geschulten Anwendung von Programmen stellt die Wahl des Verfahrens eine entscheidende Rolle, wozu sogenannte Benchmark-Beispiele beitragen. Solche werden unter anderem von der Nafems (National Agency for Finite Element Methods and Standards), der IAHR (International Association for Hydro-Environment Engineering and Research) und in Arbeitsgruppen der ICOLD (International Commission on Large Dams) für den Anwender kommentiert erarbeitet und zur Verfügung gestellt. Eine wesentliche Rolle bei der Anwendung von Rechenverfahren spielt die Ausbildung und Weitergabe von Wissen, einem Thema, zu dem vom Österreichischen Nationalkomitee für Talsperren das 8th ICOLD European Club Symposium (www.iecs2010.tugraz.at) veranstaltet wird.

Das breite Spektrum der Anwendung numerischer Verfahren wird in dieser Ausgabe durch die Beiträge eindrucksvoll dargestellt. Die Beiträge sind auch eine Aufforderung zur Weiterentwicklung der Programme, der geschulten und kritischen Anwendung dieser Verfahren für die Lösung von Ingenieuraufgaben und an die Ausbildung der Ingenieurlnnen.

\author{
Univ.-Prof. DI Dr. Gerald Zenz \\ Technische Universität Graz \\ Institut für Wasserbau und Wasserwirtschaft \\ Stremayrgasse 10/II \\ 8010 Graz \\ gerald.zenz@tugraz.at
}

